\title{
Multi-walled carbon nanotube dispersion by dispersants with different chemical structures
}

\author{
Binglin Guo ${ }^{1,}$, Xiuqi Jin ${ }^{1, b}$, Ni Liang ${ }^{1, C_{*}}$ \\ ${ }^{1}$ School of Kunming University of Science and Technology, Kunming 650500, China; \\ aguobinglin321@163.com, bdalianjinxiuqi@163.com, '751047048@qq.com
}

Keywords: CNTs, adsorption, dispersion, steric hindrance.

\begin{abstract}
Carbon Nanotubes(CNTs) are inevitably released into environment due to their wide production and application, their environmental behavior and risks are greatly controlled by their dispersion and aggregation. Polymers and surfactants were found dispersing CNTs well, however their dispersion mechanism is still not clear. Two kinds of polymers and one kind of surfactant were selected to investigate in this study, the adsorption results showed steric hindrance and electrostatic repulsion were the obstruction to adsorption and the electrostatic attraction might be the main contribution to highest adsorption of PVP. Different from adsorption, the electrostatic repulsion and steric hindrance were the essential contribution to dispersion, which in turn the highest dispersion of PSS.
\end{abstract}

\section{Introduction}

Carbon Nanotubes(CNTs) have been gaining lots of focus since Lijima discovered CNTs at first time in $1991^{[1]}$. CNTs are widely used in many areas due to their unique electrical properties, high chemical stability and larger specific surface area ${ }^{[2-4]}$, therefore CNTs were inevitably released into environment. Because CNTs have average diameters and lengths of about $1.4 \mathrm{~nm}$ and $10 \mu \mathrm{m}$ nano-scale, CNTs can go through cell membrane and damage cells ${ }^{[5]}$. CNTs may strongly interact with organic contaminants because of their high hydrophobicity and large specific surface area. Many studies have been done and found the high adsorption ability to organic contaminants ${ }^{[6-8]}$. To understand the environmental behavior of CNTs is meaningful for the reduction of CNTs pollution.

CNTs are produced in bundles or bundle aggregations, their dispersion states may be altered by adsorption to organic contaminants or dissolved organic matter(DOM). The dispersion states are controlled by at least two competitive interactions: 1) among CNTs threads, the interactions of van der Waals forces; 2) The interactions between CNTs threads and surrounding solvent moleculars. Moreover, the dispersion states of CNTs play a important role in the interactions with other contaminants that may increase the environmental risks and affect the fate of contaminants. On one hand, dispersed CNTs can expose more surface area which in turn may increase the adsorption of organic contaminants; On the other hand, CNTs dispersed through different mechanisms could influence the fate and transport of other pollutants very differently ${ }^{[9]}$. A large amount of the surface of CNTs will be wrapped by dispersants, which may provide the potential adsorption sites for other organic contaminates ${ }^{[9]}$. Besides, if CNTs are dispersed in the way of "unzipping”, more CNT surface will be available for other organic contaminates ${ }^{[6]}$. Dispersion mechanism needs to be clarified in order to understand dispersion conditions of CNTs, which can also facilitate to control the pollutant migration.

Many publications show that steric hindrance, electrostatic repulsion and micellar solubility et al. are the main dispersion mechanism of $\mathrm{CNTs}^{[10-11]}$. However, which is the most important contribution to dispersion is unclear yet. In this paper, several model compounds were selected in order to see differences in their abilities to disperse CNTs. Two kinds of polymers-Sodium polystyrene sulfonate(PSS) and polyvinylpyrrolidone(PVP) and the monomer of PSS-sodium styrene sulfonate(SS) are as the dispersants in the batch of adsorption and dispersion experiments. 


\section{Experimental}

Materials. CNTs used in the study were hydroxylized (MH), purchased from Chengdu Organic Chengdu Organic Chemistry Co., Chinese Academy of Sciences, with reported purity of $>95 \%$. The Sodium polystyrene sulfonate (PSS, $>99 \%$, average Mw 70,000) was purchased from Sigma-Aldrich Co. , polyvinylpyrrolidone (PVP, 99\%, average Mw 8,000) and styrene sulfonate (SS, 90\%) were purchased from Shanghai Aladdin Bio-Chem. Technology Co. .

Adsorption and dispersion experiments. All of the adsorption and dispersion experiments were in regular temperature of $20 \pm 2{ }^{\circ} \mathrm{C}$. PSS stock solution $(2 \mathrm{mmol} / \mathrm{L})$ was diluted by the DI water to eleven different concentrations $(0.001-0.2 \mathrm{mmol} / \mathrm{L})$, similarly PVP and SS stock solutions with the initial concentration of $1.5 \mathrm{mmol} / \mathrm{L}$ and $20 \mathrm{mmol} / \mathrm{L}$ were diluted to twelve different concentrations $(0.005-0.3 \mathrm{mmol} / \mathrm{L})$ and twelve different concentrations $(0.1-4 \mathrm{mmol} / \mathrm{L})$ respectively. $10 \mathrm{mg}$ CNTs were added to dispersant solutions with the aqueous: solid ratio of 3800:1 (w/w) in 40 $\mathrm{mL}$ glass vials with Teflon-lined screw caps, the mixed solutions were sonicated 3 min with a probe sonicater (Fisher scientific sonic dismembrator model 100, output power $=18 \mathrm{~W}$, and frequency $=$ $50 \mathrm{~Hz}$ ), then adjusted the $\mathrm{pH}$ to $7 \pm 0.2$. Samples were shaken in the dark for $72 \mathrm{~h}$ in an air-bath shaker which was long enough to reach adsorption equilibrium. Following centrifugation at 3000 $\mathrm{r} / \mathrm{min}$ for $20 \mathrm{~min}$ and the supernates were used to measure the dispersion by Agilent 8453 spectrometer with the absorbance at $800 \mathrm{~nm}$ and the Zeta-potentials using Zetasizer (90 Plus Zeta, Brookhaven) with a folded capillary cell. And after adsorption equilibrium the $\mathrm{pH}$ didn't change much, $\mathrm{pH}$ values were between 5.0 and 6.3. After filtration with $0.45 \mu \mathrm{m}$ membrane (Shanghai Anpel Scientific Instrument Co. Ltd.), the supernates were used to measure the aqueous concentrations of PSS, PVP and SS at the absorbance of $256 \mathrm{~nm}, 194 \mathrm{~nm}$ and nm respectively with the UV-vis.

Data analysis. Adsorption isotherms were fitted utilizing the Freundlich model based on the least squares minimization with SigmaPlot 10.0.

$$
\lg Q_{\mathrm{e}}=\lg K_{\mathrm{F}}+n \cdot \lg C_{\mathrm{e}}
$$

Where $Q_{\mathrm{e}}(\mathrm{mg} / \mathrm{kg})$ and $C_{\mathrm{e}}(\mathrm{mg} / \mathrm{L})$ are the equilibrium solid-phase and aqueous-phase concentrations respectively, $K_{\mathrm{F}}\left[(\mathrm{mg} / \mathrm{Kg}) /(\mathrm{mg} / \mathrm{L})^{\mathrm{n}}\right], n$ is the nonlinearity factor.

\section{Results and discussion}

Adsorption.

Table 1 Fitting results of adsorption isotherms of three dispersants

\begin{tabular}{ccccc}
\hline & \multicolumn{3}{c}{ Freundlich Parameters } & $K_{\mathrm{d}}\left(\mathrm{L} \cdot \mathrm{g}^{-1}\right)$ \\
\cline { 2 - 5 } Dispersants & $r^{2}{ }_{\text {adj }}$ & $K_{\mathrm{F}}$ & $n$ & $\lg C_{\mathrm{e}}=2.7\left(\mathrm{mg} \cdot \mathrm{L}^{-1}\right)$ \\
& & 1224.334 & 0.698 & \\
\hline SS & 0.982 & 1439.130 & 0.720 & 251.779 \\
PVP & 0.994 & 637.529 & 0.779 & 161.261 \\
\hline
\end{tabular}




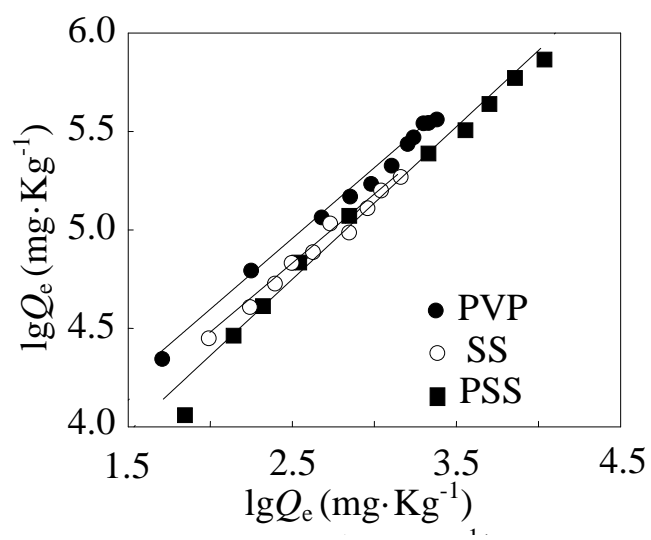

Fig 1 Adsorption isotherms of three dispersants on CNTs
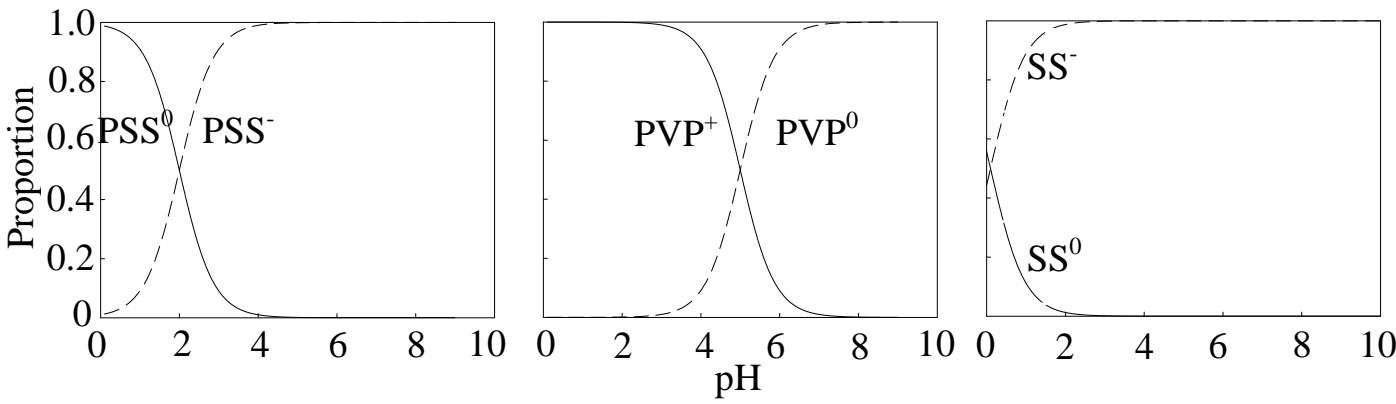

Fig 2 Species distributions of three dispersants at different pHs

Freundlich mode was selected to fit adsorption isotherms, the fitting results were presented in table 1. In table 1, three $r^{2}$ adj values were all above 0.98, that meant Freundlich mode could fit the adsorption isotherms well. The $K_{\mathrm{F}}$ values followed the decreasing order by PVP, SS and PSS, it revealed that PVP adsorbed on CNTs was higher than SS and PSS. The $n$ values were all close to 1, which revealed the adsorption isotherms were linear, suggesting the highly homogeneous distribution of adsorption sites on CNT surface ${ }^{[12]}$. The sorption coefficient $K_{d}$ followed the order of PVP > SS > PSS, suggesting the adsorption of PVP on CNTs was higher than SS and PSS. The mechanism of different adsorption results were written in detail later.

As shown in Fig 1, the adsorption of PVP on CNTs was higher than PSS and SS, and the adsorption of SS was higher than PSS. The species distribution of three dispersants were calculate ed shown in Fig 2, meant that in experiment pHs, the distribution proportion of SS negative specie was almost 100\%, for PSS was above 99\% and PVP was between 50\%-96\% respectively. The CNTs selected in the study was hydroxylized, the surface was highly negative charged under experiment pHs. The electrostatic interaction between CNT bundles and PVP molecular was lower than PSS and SS, the strong repulsion made PSS and SS molecular difficult to approach to CNT surface. As for PSS and SS, they were all highly negative charged, so the electrostatic interaction was not the cause of different adsorption results. On one hand, the average molecular weight of PSS was large compared with SS; On the other hand, PSS was difficult to access to the CNT surface because of the strong steric hindrance due to its huge steric structure.

Dispersion. Fig 3 showed the stabilization of CNTs in three dispersant solutions, UV-vis spectrophotometry at $800 \mathrm{~nm}$ absorbance were used to estimate the dispersion of CNTs. CNTs were dispersed in three kinds of solutions was attributed to the wrapping of dispersant chain around the CNT bundles. The dispersion of CNTs improved with the increasing three dispersants at low concentrations. However, as for two polymers CNTs dispersion decreased with the increasing of their adsorption at high concentrations. At low concentrations, 1) improved adsorption provided increasing steric hindrance, which was also founded by several studies, Liping Zhao founded that the CNT suspension with copolymer was mainly attributed to the steric stabilization, the polymers could conquer the van der Waal's through steric hindrance and helped to separate CNT bundles from each other ${ }^{[13]}$; 2) The increasing electrostatic repulsion played a important role in dispersing 
CNTs, which could be inferred from zeta potential results shown in Fig 4, the zeta potential rose with the increasing of adsorption, which meant the increased negatively charged surface by the adsorption of dispersants increased the electrostatic repulsion between CNTs bundles and the dispersion was facilitated. However, CNT dispersion trends of PSS and PVP were different from SS at high concentrations. The dispersion of CNTs decreased with the increasing adsorption. The mechanism was speculated where one polymer molecule might link two or more nanotubes should be responsible for their aggregation, Alla L. Alpatova et al. also found that the aggregation of CNTs at high PVP concentrations ${ }^{[14]}$. The above discussion showed that steric hindrance and electrostatic repulsion were essential contribution to dispersion of CNTs in this study.

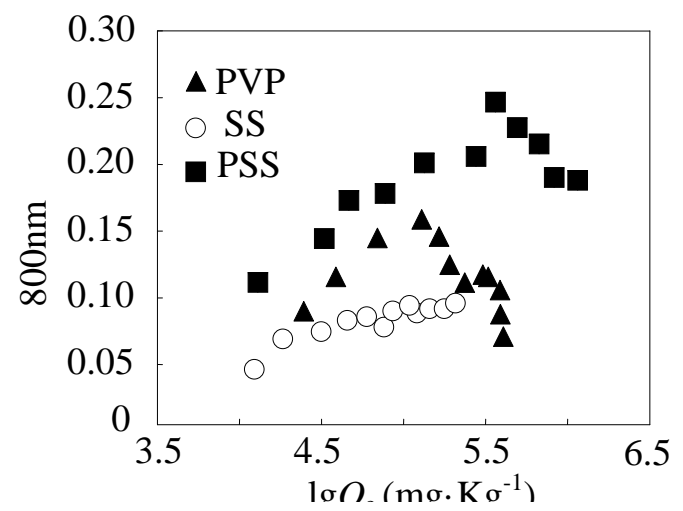

Fig 3 Dispersion of CNTs by three dispersants

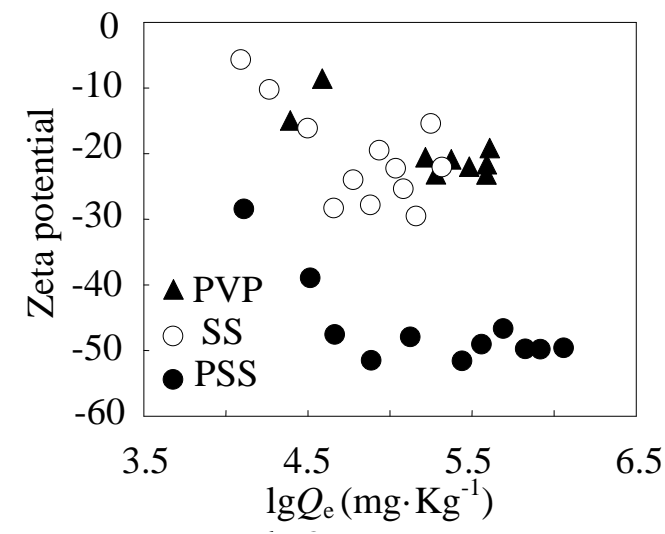

Fig 4 The Zeta potential of CNTs surface at different concentration of three dispersants

The dispersion of CNTs followed the decreasing order by PSS, PVP and SS, it meant the repulsion between CNT bundles increased. It's easy to observe that the repulsion rose with the increasing of the length of grafted chains. The surface coveraged with dispersant chains could provide strong steric hindrance which in turn hindered bundles' aggregation, so CNTs dispersed better in PSS solution than others due to its longest molecule chain, same results were founded by Rina Shvartzman-Cohen et al. , they also found the increasing of length of the grafted chain strengthen the repulsion between CNT bundles ${ }^{[15]}$. Furthermore, observing in Fig 4, the zeta potential results showed PSS coated CNTs had the highest Zeta potential value that reflected highest electrostatic repulsion, so the highest electrostatic repulsion and the strongest steric hindrance made highest dispersion in PSS solution. Although the zeta potential of PVP was same as SS, its dispersion was higher than SS, the longer molecule chain provided stronger steric hindrance to facilitate the dispersion. Same as previous studies, electrostatic repulsion and steric hindrance were essential parts in dispersion of CNTs and polymers complex systems ${ }^{[14]}$.

\section{Summary}

In this study, we found that due to the different length of molecule chains and the electrostatic properties of different dispersants, their adsorption results on CNTs were different, strong 
electrostatic repulsion and strong steric hindrance hinder the adsorption of PSS and SS. As for dispersion, on the contrary, the electrostatic repulsion and steric hindrance were the essential contribution to CNT dispersion, which got CNTs the highest dispersion in PSS.

\section{Reference}

[1] Iijima S. Helical microtubules of graphitic carbon[J]. nature, 1991, 354(6348): 56-58.

[2] Tang X, Bansaruntip S, Nakayama N, et al. Carbon nanotube DNA sensor and sensing mechanism[J]. Nano Letters, 2006, 6(8): 1632-1636.

[3] Clayton L N M, Sikder A K, Kumar A, et al. Transparent Poly (methyl methacrylate)/Single-Walled Carbon Nanotube (PMMA/SWNT) Composite Films with Increased Dielectric Constants[J]. Advanced Functional Materials, 2005, 15(1): 101-106.

[4] Frackowiak E, Beguin F. Electrochemical storage of energy in carbon nanotubes and nanostructured carbons[J]. Carbon, 2002, 40(10): 1775-1787.

[5] Zhu Y, Zhao Q, Li Y, et al. The interaction and toxicity of multi-walled carbon nanotubes with Stylonychia mytilus[J]. Journal of nanoscience and nanotechnology, 2006, 6(5): 1357-1364.

[6] Pan B, Xing B. Adsorption mechanisms of organic chemicals on carbon nanotubes[J]. Environmental Science \& Technology, 2008, 42(24): 9005-9013.

[7] Sheng G D, Shao D D, Ren X M, et al. Kinetics and thermodynamics of adsorption of ionizable aromatic compounds from aqueous solutions by as-prepared and oxidized multiwalled carbon nanotubes[J]. Journal of hazardous materials, 2010, 178(1): 505-516.

[8] Wang X, Liu Y, Tao S, et al. Relative importance of multiple mechanisms in sorption of organic compounds by multiwalled carbon nanotubes[J]. Carbon, 2010, 48(13): 3721-3728.

[9] Zhang D, Pan B, Cook R L, et al. Multi-walled carbon nanotube dispersion by the adsorbed humic acids with different chemical structures[J]. Environmental Pollution, 2015, 196: 292-299.

[10] Jiang L, Gao L, Sun J. Production of aqueous colloidal dispersions of carbon nanotubes[J]. Journal of Colloid and Interface Science, 2003, 260(1): 89-94.

[11] Yang K, Yi Z L, Jing Q F, et al. Sonication-assisted dispersion of carbon nanotubes in aqueous solutions of the anionic surfactant SDBS: The role of sonication energy[J]. Chinese Science Bulletin, 2013, 58(17): 2082-2090.

[12] Wang Z, Yu X, Pan B, et al. Norfloxacin sorption and its thermodynamics on surface-modified carbon nanotubes[J]. Environmental Science \& Technology, 2009, 44(3): 978-984.

[13] Zhao L, Gao L. Stability of multi-walled carbon nanotubes dispersion with copolymer in ethanol[J]. Colloids and Surfaces A: Physicochemical and Engineering Aspects, 2003, 224(1): 127-134.

[14] Alpatova A L, Shan W, Babica P, et al. Single-walled carbon nanotubes dispersed in aqueous media via non-covalent functionalization: effect of dispersant on the stability, cytotoxicity, and epigenetic toxicity of nanotube suspensions[J]. water research, 2010, 44(2): 505-520.

[15] Shvartzman-Cohen R, Nativ-Roth E, Baskaran E, et al. Selective dispersion of single-walled carbon nanotubes in the presence of polymers: the role of molecular and colloidal length scales[J]. Journal of the American Chemical Society, 2004, 126(45): 14850-14857. 